\title{
Stimulus Component Independence ${ }^{1}$
}

\author{
EDWIN MARTIN \\ University of Michigan, Ann Arbor, Michigan 48104
}

\begin{abstract}
After paired-associate learning to one of three degrees of learning with compound stimuli, the stimulus components were presented in isolation and $S$ required to respond with the other components and the response. Response recall failure precluded recall of other components. This result was shown not traceable to a small number of such events, to item selection, or to the possibility that $S$ forms intercomponent associations only after forming component-response associations. When the response was recalled, recalls of other components were frequent but stochastically independent of each other. Nevertheless, standard stimulus selection phenomena occurred. These analyses deny the general assumption that stimulus components become interassociated during paired-associate learning.
\end{abstract}

Richardson's (1971) excellent assessment of stimulus selection in paired-associate learning contains a short section entitled What is learned? That section is actually a subsection under the more general topic of pairedassociate learning with compound stimuli. In the subsection, Richardson reports that there is evidence for association formation among the components of a compound stimulus. The purpose of the present paper is two-fold: to argue that conclusions about association formation among stimulus components cannot be drawn from the studies ordinarily cited, and to present the results of a standardly conducted but specially analyzed stimulus selection experiment that demonstrates complete absence of componentcomponent association formation over several degrees of learning and over several response anticipation intervals.

The Steiner and Sobel (1968, Exp. II) study represents perhaps the most ingenious of the relevant experimental designs. The stimuli were eight word-color combinations; the

\footnotetext{
${ }^{1}$ This work was supported by the Advanced Research Projects Agency, Department of Defense, and monitored by the Air Force Office of Scientific Research, under Contract No. AF 49(638)-1736 with the Human Performance Center, Department of Psychology.
}

responses, four single-digit numbers. Each response was paired with two completely distinct stimulus compounds. In the test phase, after paired-associate learning, the $S$ was faced with matching each of the word components with one of three color patches. In Type 1 tests, the three colors were $(a)$ the color that went with the word in training, (b) the color that did not go with that word but instead went with the other word that had the same digit response, and $(c)$ a color that was incorrect on either count. These three colors thus had as possible bases for matching selection (a) either a direct intercomponent (S-S) association or an indirect S-R-S chain of associations, $(b)$ an indirect $\mathrm{S}-\mathrm{R}-\mathrm{S}$ chain of associations originating with the wrong color but passing through the correct common response, or $(c)$ neither. Type 2 tests pitted $a$ matches against $c$ matches. Type 3 tests pitted $b$ matches against $c$ matches. All of this was handsomely done within $S$ s. The results of the matching tests were analyzed by subtracting one observed proportion from another. Thus, for example, subtracting the Type $1 b$ observed proportion (errors which could involve only an indirect S-R-S chain) from the Type $1 a$ observed proportion (correct matches which could involve either $\mathrm{S}-\mathrm{S}$ or S-R-S matching) was the source of evidence 
that S-S matching indeed occurred. The authors concluded that intercomponent (S-S) associations had been formed during learning.

But there is a very good reason for discounting this conclusion. For those match alternatives wherein direct $\mathrm{S}-\mathrm{S}$ matching was possible, Type $1 a$, there were perforce two sources of origin for a correct match, namely, both the word and the color. Type $1 b$ match alternatives involved only one source of origin, namely, the color. The procedure of subtracting away the rate of matching based only on backward color-to-response-to-word errors from that based possibly on both S-S and S-R-S matching is not a satisfactory correction because it assumes a strict linear combination of the effects of two associations that are assumed to be noninteractive. What is required is a way to preclude any possible mediation through the response member; in doing so, one removes the possibility of facilitative interaction between corresponding associations that are oppositely directed.

As distinct from an experimental design problem, there is the other case where the phrasing of the research problem works against thinking of certain analyses of the data. An example of this is the Postman and Greenbloom (1967) report, which verbal learning theorists tend to classify as supportive of the idea of intercomponent association formation. Traditionally, after paired-associate learning has been taken to a criterion, a stimulus-components transfer task is introduced. Each component is presented alone and the proportion response recalls observed. These observed proportions are then used to index the degree of component selection. Postman expresses his reservation about such results as follows:

It is not known how frequently responses made to a single letter were mediated by recall of either or both of the remaining letters. To the extent that mediation occurs, correct recalls do not in fact reflect associations to single letters [p. 91-92].

This introductory statement of a proper reservation entails, however, a clear assumption of intercomponent association formation. The subsequent results show that when a single stimulus component is presented, the probability of response recall is directly related to success in recalling the other, not-presented stimulus components. This result is certainly compatible with the presumptive introductory idea of intercomponent association formation; but it is also compatible with the opposite idea, namely, that recall of the other, not-presented stimulus components was mediated through the response member. Conditionalizing stimulus-component recall on response recall was not considered.

If original paired-associate learning involves a compound stimulus, $A=\left\{A_{1}, A_{2}\right\}$, and a response, $B$, then the two general inferential faults in concluding for intercomponent association formation may be summarized as follows:

1. The assumption that associations $A_{i} \rightarrow B$ and $B \rightarrow A_{i}$ are noninteractive and hence that their individual effects, as represented by a posteriori probabilities (unconditional relative frequencies), can be added or subtracted algebraically.

2. The assumption that an $A_{i} \rightarrow A_{\jmath}$ association exists, to the end that the $A_{i} \rightarrow B \rightarrow A_{j}$ mediational possibility is not examined.

These two faults were exemplified above by the Steiner and Sobel (1968) and the Postman and Greenbloom (1967) papers, respectively, but apply to the published literature in general. The remedy for both of these problems is to eliminate somehow any possible action of the $B$ response member in tests for $A_{\imath} \rightarrow A_{j}$ intercomponent associations.

The experiment to be reported is an extension of an earlier experiment by Wichawut and Martin (1970). In reporting the results of the present experiment, we shall incorporate new analyses of the earlier experiment. ${ }^{2}$

\footnotetext{
2 The present experiment was conducted and analyzed by Stephen A. Mackay. The additional analyses of the Wichawut and Martin (1970) experiment incorporated into this report were carried out by Chaiyaporn Wichawut.
} 
Since the experimental procedure itself is traditional and noninnovative, what is of interest are the analyses. These were directed to a single question: When the possibly mediating action of the response is eliminated, is there any residual evidence for interassociation among the stimulus components during learning?

\section{MeThoD}

\section{Design and Materials}

Four groups of eight $S \mathrm{~s}$ each learned, by the anticipation procedure, a list of eight paired-associates to a criterion of $8 / 8$ or $8 / 8+8$ with an anticipation interval of either $1 \mathrm{sec}$ or $4 \mathrm{sec}$. The design was thus a completely between-subjects $2 \times 2$ factorial with two degrees of learning and two anticipation intervals. The Wichawut and Martin (1970) experiment utilized a 2-sec anticipation interval and three degrees of learning, $8 / 8,8 / 8+8$, and $8 / 8+16$.

Each stımulus was a triad of unrelated (according to local, consensus) four-letter words. The response member was a single four-letter word, unrelated (also according to local consensus) to any of the other words in the list. Example: FUND SILK DOVE as a stimulus; WHIP as a response. Hereinafter, the three stimulus words will be labelled $A_{1}, A_{2}$, and $A_{3}$, respectively, from left to right.

After learning, the $S \mathrm{~s}$ were shown all 32 words from the list (24 stimulus words, 8 response words). The words were presented singly, one at a time, in a 32-page booklet with positional blanks for the three words not presented on that page. The order of occurrence of the words in the booklets was random and varied from $S$ to $S$. The $S$ s were to write in the missing words.

\section{Procedure}

The learning lists were presented etther at a $1: 2$-sec or a $4: 2-\mathrm{sec}$ rate on a Stowe memory drum. The $S$ attempted to respond verbally with the correct response before it was shown to him. The stimuli were not responded to overtly. The intertrial interval was $4 \mathrm{sec}$. There were eight different orders of pair presentation. Approximately $1 \mathrm{~min}$ elapsed between meeting the learning criterion and beginning the recall test, during which time the recall test instructions were given.

In the recall test, $S$ was instructed to work his way through the booklet at his own rate, writing in all missing words, but not turning back to previous pages. He was explicitly allowed to guess.

\section{Subjects}

The $S$ s were undergraduate women enrolled in summer school in the University of Michigan. They volunteered for paid participation and had no previous experience in experiments of this sort. They were tested individually.

\section{RESUlTs AND DisCusSION}

From this point onward we shall incorporate the results of the Wichawut and Martin (1970) experiment. That experiment was identical to the present one in all respects save the anticipation interval, which was $2 \mathrm{sec}$, the additional degree of overlearning, $8 / 8+16$, and the $S$ population, regular fall-term students. Throughout, the earlier results can be identified by the 2-sec anticipation interval or the $8 / 8+16$ learning criterion.

In Table 1 are shown the mean number trials to a criterion of $8 / 8$, irrespective of whether either 8 or 16 additional learning trials were imposed. These means range disgracefully from 9.5 to 27.3 . No analysis of variance is necessary. On the other hand, three points: All $S$ s reached their assigned criterion; all the analyses that follow are conditional against other aspects of performance; and, after all, these learning-rate differences had no discernible effect on the pattern of recall test results.

There is a single critical analysis of the recall test protocols. The results of this analysis are shown in Fig. 1. Consider first the upper three curves. Given that stimulus word $A_{i}$ (collapsed over $i=1,2,3$ ) was the presented test word and that the response was recalled to that stimulus word, the $S$ could recall either both, one, or neither of the two remaining stimulus words. The upper curves show the proportion of these opportunities that resulted in recall

\section{TABLE 1}

Mean Trials to $8 / 8$ Learning Criterion

\begin{tabular}{cccc}
\hline $\begin{array}{c}\text { Anticipation } \\
\text { interval (sec) }\end{array}$ & $8 / 8$ & $8 / 8+8$ & $8 / 8+16$ \\
\hline 1 & 22.4 & 15.0 & - \\
2 & 11.8 & 12.5 & 9.5 \\
4 & 27.3 & 23.4 & - \\
\hline
\end{tabular}


of at least one of the other two remaining stimulus words, as a function of degree of learning, with anticipation interval $(1,2,4 \mathrm{sec})$ as the parameter. Evidently, recall of other stimulus words increased regularly both with degree of learning and with length of anticipation interval, given, that is, that the response was also recalled.

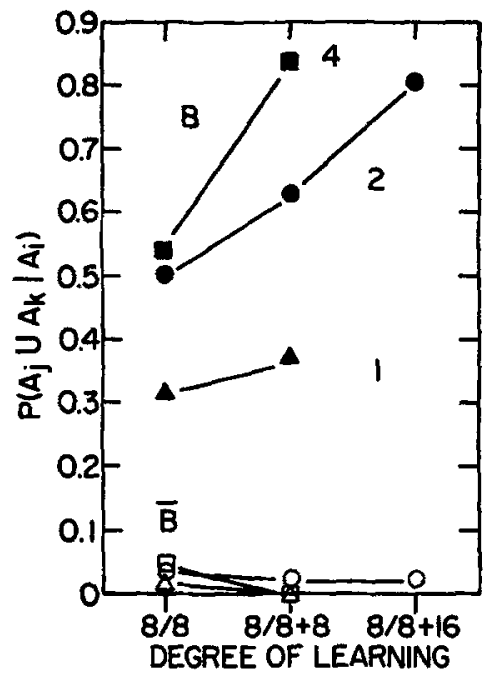

FIG. 1. Proportion times at least one stimulus word $\left(A_{j} \cup A_{k}\right)$ recalled given stimulus word $A_{i}$ presented and response was (B, upper curves) and was not $(\bar{B}$, lower curves) recalled, as a function of degree of learning, with anticipation interval as the parameter.

The lower three curves in Fig. 1 depict the exact same proportions, except that in these cases the response was not recalled. These curves are very close to zero. What they mean is that if $A_{i}$ was the presented stimulus word and if $S$ could not recall the response, then he could not recall any of the other stimulus words. These lower curves are not based on small numbers. For example, the three opencircle points (the 2-sec anticipation interval) are based on 410,333 , and 241 response-recall failures, respectively, from left to right. [The maximum possible number of responserecall failures or successes is ( 3 stimulus word positions $) \times(8$ stimuli $) \times(32 \mathrm{Ss})=768$ for this particular curve.]
The results shown in Fig. 1 are not attributable to item selection on the part of the $S \mathrm{~s}$, where by item selection is meant the sort where $S$ does not work very hard to learn some of the pairs. Consider all recall test events wherein stimulus word $A_{i}$ was presented and the response was not recalled. Taking note of those responses in relation to the words $A_{i}$ that failed to elicit them, we can determine the probability that when that response was itself presented it elicited at least one of the stimulus words $A_{j}$ or $A_{k}$ different from $A_{i}$. These probabilities are shown in Table 2 . They are of respectable magnitude and an orderly function of degree of learning. Also in Table 2 is shown the probability that such a response elicited backwardly, either alone or in conjunction with other stimulus words, the stimulus word that failed to elicit it. These probabilities are very small. Thus when a presented stimulus word failed to elicit its response it was because $S$ did not use that particular stimulus word as a functional cue; instead he used one or the other or both of the other two words.

But an objection can be raised to these results, an objection, however, whose test is straightforward. ${ }^{3}$ Perhaps $S$ selected a single

\section{TABLE 2}

For $B$ Responses Not Recalled to COMponent $A_{i}$, PROPORTION EITHER $A_{f}$ OR $A_{k}$ OR BOTH RECALLED $\left(A_{j} \cup A_{k}\right)$ AND Proportion $A_{i}$ Recalled When Response $B$ Presented

\begin{tabular}{lccc}
\hline & \multicolumn{3}{c}{ Degree of learning } \\
\cline { 2 - 4 } $\begin{array}{c}\text { Condition } \\
\text { statistic }\end{array}$ & $8 / 8$ & $8 / 8+8$ & $8 / 8+16$ \\
\hline $1 \mathrm{sec}$ & & & \\
$A_{j} \cup A_{k}$ & .73 & .75 & - \\
$A_{t}$ & .04 & .05 & - \\
$2 \mathrm{sec}$ & & .89 & .97 \\
$A_{j} \cup A_{k}$ & .76 & .07 & .12 \\
$A_{i}$ & .05 & .90 & - \\
$4 \mathrm{sec}$ & .64 & 0 & - \\
$A_{j} \cup A_{k}$ & .02 & 0 & \\
$A_{t}$ & .05 &
\end{tabular}

${ }^{3}$ My appreciation to Robert A. Bjork for this point. 
stimulus word and associated to it the correct response word. Having done this, $S$ then, and not until then, began to form associations among the several stimulus words. Were this the true course of events, then failure to recall the $B$ response to the presented stimulus component $A_{i}$ would necessarily result in inability to recall the remaining two stimulus components, $A_{j}$ and $A_{k}$, and hence the pattern of results shown in Fig. 1. Thus if intercomponents association formation occurred only after an $A_{i} \rightarrow B$ association had been completed, the upper curves in Fig. 1 would represent pairs for which the initial $A_{i} \rightarrow B$ stage was completed before testing, and the lower curves would represent pairs for which the initial $A_{i} \rightarrow B$ stage was not completed before testing.

But this two-stage possibility cannot be accurate. Consider the pattern of results shown in Table 2. Whenever a given stimulus word $A_{i}$ failed to elicit the correct $B$ response, that $B$ response nevertheless elicited at least one of the other two stimulus words with the $A_{j} \cup A_{k}$ probabilities shown in that table, indicating that for most pairs the initial $A_{i} \rightarrow B$ stage was complete for at least one $A_{i}$. Thus failure or absence of a given $A_{i} \rightarrow B$ association meant merely that $A_{i}$ was not utilized, that instead some other component was utilized. Failure or absence of a given $A_{t} \rightarrow B$ association cannot be used as evidence that an initial component-response stage had not been completed.

Also relevant as evidence against the twostage possibility just discussed, but of interest in its own right, is the result of the following test for intercomponent independence. Consider those recall test events where stimulus word $A_{i}$ was presented and the corresponding response was recalled, that is, those recall test events wherein a stimulus word was presented and $S$ had any hope of recalling any of the other stimulus words. As shown by the upper curves in Fig. 1, there was frequent incidence of recall of stimulus words other than presented $A_{i}$. If $A_{j}$ and $A_{k}$ are the other two stimulus words, then we can easily tally the frequency of recall of $A_{j}$ and $A_{k}$ and calculate the unconditional probabilities $P\left(A_{\jmath}\right)$ and $P\left(A_{k}\right)$. If it is true that $A_{j}$ and $A_{k}$ are recalled independently of each other, then the product $P\left(A_{J}\right) P\left(A_{k}\right)$ should predict exactly the relative frequency of joint recalls of $A_{j}$ and $A_{k}$. That is, if stimulus word independence is the case, then the frequencies of recall of $A_{j}$ and $A_{k}$ should sustain the equality

$$
P\left(A_{j}\right) P\left(A_{k}\right)=P\left(A_{j} \& A_{k}\right) \text {. }
$$

For each $A_{i}(i=1,2,3)$, each anticipation interval $(1,2,4 \mathrm{sec})$, and each degree of learning $(8,8 / 8+8,8 / 8+16), P\left(A_{j}\right), P\left(A_{k}\right)$, and $P\left(A_{j} \& A_{k}\right)$ were calculated. Fig. 2 is a plot of $P\left(A, \& A_{k}\right)$ against the product $P\left(A_{j}\right) P\left(A_{k}\right)$. Which stimulus word $\left(A_{1}, A_{2}, A_{3}\right)$ and which anticipation interval $(1,2,4 \mathrm{sec})$ determine a given point is shown in the legend at the top of the figure. The several occurrences of a particular type of point are the several degrees of learning for that combination of stimulus word and anticipation interval. These several occurrences are properly ordered in all cases.

Verification of the independence identity expressed in the equation above requires all the points to fall on the dashed diagonal line in Fig. 2. The points are very close. To the extent that they are acceptably coincidental with the diagonal line, we can argue that recall of stimulus word $A_{f}$ in no way determined recall of stimulus word $A_{k}$. This, together with the analyses showing that $S$ could not recall either $A_{j}$ or $A_{k}$ when $A_{i}$ was presented except via mediation through response $B$, means that the components of the compound stimuli acted independently of each other and that the responses developed parallel, noninteractive dependencies (associations) with these independent components during learning.

There are two related reservations worth noting. In Fig. 1, the three lower curves are close to zero, but not exactly zero. Accordingly, a case can be made that the stimulus words were not entirely free of interassociations. 
Similarly, in Fig. 2 the points are close to the diagonal line, but not exactly coincidental. Accordingly, a case can be made that the stimulus words did not act entirely independently. My inclination, however, is to conclude that local consensus on the a priori unrelatedness of the stimulus words was deficient in

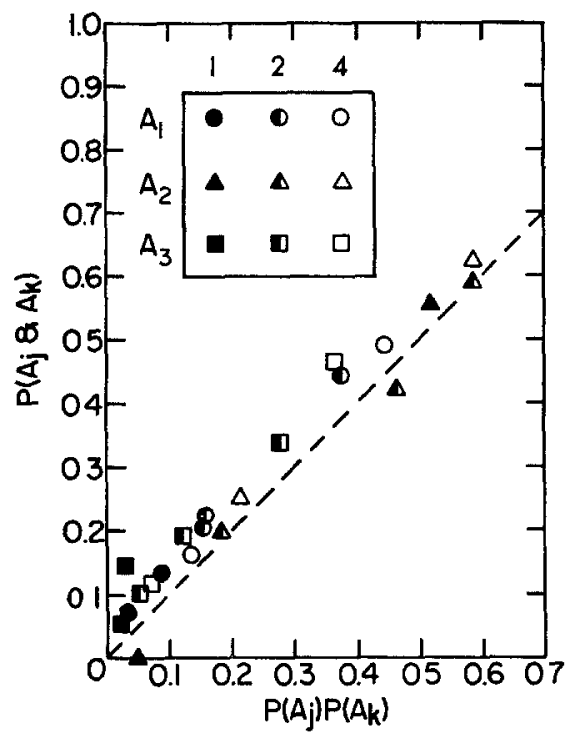

FIG. 2. Plot of proportion joint recalls of stimulus words $A_{j}$ and $A_{k}$ given stimulus word $A_{i}$ presented and the response recalled, against the product of the corresponding unconditional proportions, for presented $A_{1}, A_{2}, A_{3}$ and anticipation intervals $1,2,4 \mathrm{sec}$. Multiple points of the same kind are for different degrees of learning.

accuracy, at least in so far as that accuracy completely encompassed any relatedness gleaned by a (very) few $S$ s. That it is indeed a matter of infrequent $a$ priori relatedness as opposed to intercomponent association formation during learning is clear from the fact that departures from zero of the lower three curves in Fig. 1 and departures from the diagonal line in Fig. 2 are in no way related to degree of learning.

That the word components of a compound stimulus formed parallel and independent associations with the prescribed response does not, of course, mean that the usual phenomena of stimulus selection did not occur. In general, $A_{3}$ was the most effective stimulus word, $A_{1}$ the next most effective, and $A_{2}$ the least most effective. The preceding independence results supplement this standard fact with the additional fact that, for example, the frequent selection of $A_{3}$ did not causally determine, on an intercomponent basis, the next-most-frequent selection of $A_{1}$.

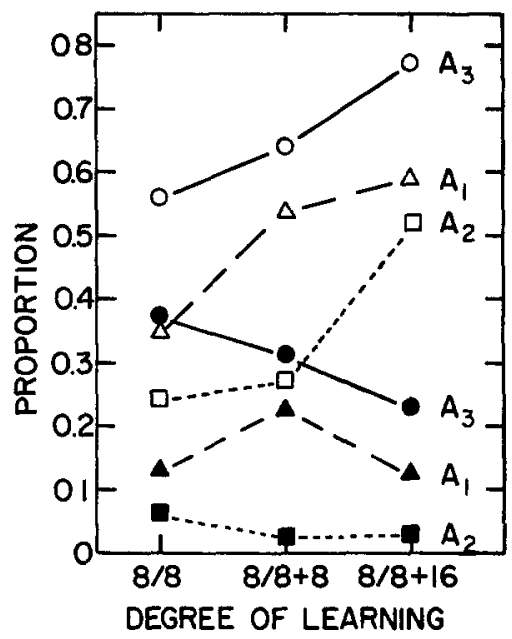

FIG. 3. Proportion recall of stimulus words $A_{1}$, $A_{2}, A_{3}$ as a function of degree of learning. Upper curves: All recalls of $A_{i}$. Lower curves: Recalls of $A_{i}$ only.

Plotted in Fig. 3 are the proportion times $A_{\boldsymbol{t}}$ was recalled when the response term was the presented word, as a function of degree of learning, collapsed over anticipation interval. The upper, open-figure curves are the proportion recalls of $A_{i}$ whether alone or in conjunction with other stimulus words. The lower, filled-figure curves are the proportion recalls of $A_{\imath}$ alone, that is, only $A_{i}$ with no recall of either of the other two stimulus words.

Several features of Fig. 3 are worth noting. Although $A_{2}$ was seldom recalled alone, it was often recalled in conjunction with the other stimulus words $\left(A_{1}\right.$ and $\left.A_{3}\right)$. Overlearning increased selection of $A_{2}$ in an independent, additive way. Over degrees of learning, recall of $A_{3}$ alone decreased regularly (lower curve 
with filled circles). This, together with the increasing unconditional frequency of recall of $A_{3}$ (upper curve with open circles), again indicates that $S$ s were adding more words to their effective stimulus as degree of learning increased. Finally, the relative effectiveness of the three words (the upper three curves in Fig. 3) remained more or less constant over the three degrees of learning.

These facts mean that there was a definite pattern of stimulus word selection and that with overlearning the $S$ s picked up additional stimulus words as effective cues. The earlier analyses show that these stimulus words did not interassociate with each other, that they acted independently of each other. The implication of these results is that previous conclusions of intercomponent association formation may be unjustified. This is not to deny that intercomponent associations can exist or perhaps even be formed during learning, rather to argue that since it is easy to test for such associations, say nothing of exhibit their absence, one might well wonder about the validity of assumptions of their reality.

\section{REFERENCES}

Postman, L., \& Greenrloom, R. Conditions of cue selection in the acquisition of paired-associate lists. Journal of Experimental Psychology, 1967, 73, 91-100.

RichaRdson, J. Cue effectiveness and abstraction in paired-associate learning. Psychological Bulletin, $1971,75,73-91$.

Steiner, T. E., \& Sober, R. Intercomponent association formation during paired-associate learning with compound stimuli. Journal of Experimental Psychology, 1968, 77, 275-280.

Wichawut, C., \& Martin, E. Selective stimulus encoding and overlearning in paired-associate learning. Journal of Experimental Psychology, $1970,85,383-388$.

(Received July 3, 1971) 